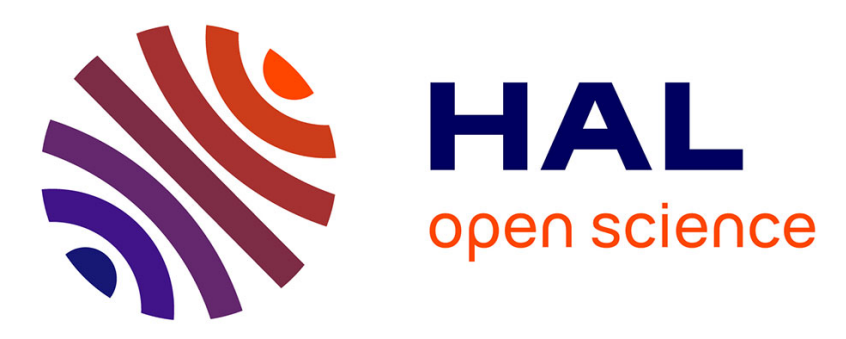

\title{
Simulation of disordered systems of cylinders. I. geometrical behaviour
}

\author{
S. Roux, D. Stauffer, H.J. Herrmann
}

\section{To cite this version:}

S. Roux, D. Stauffer, H.J. Herrmann. Simulation of disordered systems of cylinders. I. geometrical behaviour. Journal de Physique, 1987, 48 (3), pp.341-345. 10.1051/jphys:01987004803034100 . jpa00210448

\section{HAL Id: jpa-00210448 https://hal.science/jpa-00210448}

Submitted on 1 Jan 1987

HAL is a multi-disciplinary open access archive for the deposit and dissemination of scientific research documents, whether they are published or not. The documents may come from teaching and research institutions in France or abroad, or from public or private research centers.
L'archive ouverte pluridisciplinaire HAL, est destinée au dépôt et à la diffusion de documents scientifiques de niveau recherche, publiés ou non, émanant des établissements d'enseignement et de recherche français ou étrangers, des laboratoires publics ou privés. 
Classification

Physics Abstracts

$05.40-46.30 \mathrm{C}$

\title{
Simulation of disordered systems of cylinders. I. Geometrical behaviour
}

\author{
S. Roux $\left({ }^{1}\right)$, D. Stauffer $\left({ }^{1,2,3}\right)$ and H. J. Herrmann $\left({ }^{2}\right)$ \\ (1) LHMP UA857, ESPCI, 10, rue Vauquelin, 75231 Paris Cedex 05, France \\ $\left.{ }^{2}\right)$ SPhT, CEN Saclay, 91191 Gif-sur-Yvette, France \\ $\left({ }^{3}\right)$ Permanently at: Inst. Theor. Phys., University, 5000 Köln 41, F.R.G.
}

(Reçu le 28 juillet 1986, révisé le 10 octobre, accepté le 24 octobre 1986)

\begin{abstract}
Résumé. - Nous discutons l'empilement de cylindres parallèles dont le rayon fluctue peu. Le sous-réseau des contacts consiste en des structures arborescentes auto-similaires. L'expansion moyenne de l'empilement et la distribution des distances entre cylindres sont aussi présentées.
\end{abstract}

\begin{abstract}
We discuss the piling of parallel cylinders with weakly fluctuating radii. The network of contacts consists of self-similar tree-like patterns. The mean expansion of the piling and the distribution of distances between adjacent cylinders are also investigated.
\end{abstract}

\section{Introduction.}

Granular, composite random materials and their mechanical or electrical properties are of great practical importance. Several theoretical models for the elastic properties of diluted disordered systems have been recently introduced; see e.g. reference [1]. Experimentally, one way to study a simplified model system is to pack together many parallel cylinders of different elastic modulus, to compress the system, and to measure the relation between pressure and contraction. In such an experiment, a strongly non-linear relation was observed [2] which differed drastically from the behaviour of an individual pair of cylinders (Hertz law [3]). The aim of this and a companion article [4] is to elucidate theoretically the possible reasons for this behaviour. Reference [5] discusses analogous nonlinear effects in electrical disordered system.

In a simulation of disordered materials, one has to take into account that real cylinders are not ideal ; they may not be exactly circular in cross-section, their centre line may not be entirely straight, their surface may be rough, and their radii may not be all identical. In this work and in the companion paper [4] we take into account only this last effect since it suffices already to give a macroscopic elastic behaviour drastically different from the microscopic law, in agreement with experiment [2]. We do not claim that these radius fluctuations are the only possible explanation for this experimental observation.
When small cylinders of equal radii are packed together orderly, they form a triangular lattice. When their radii fluctuate slightly, the triangular lattice is also distorted. To get the resulting expansion in the limit of zero force is quite difficult both in experiments [2] and in our computer simulations [4]. Thus the first part describes a different attempt to calculate the purely geometrical effects in zero force. This method of nearly ordered packing is, of course, different from the problem of strongly disordered packing (see e.g. Ref. [6]) which is less relevant to our issue.

\section{The algorithm.}

Let us consider a pile of cylinders which have radii $0.5+\varepsilon$ inside a rectangular box, $\varepsilon$ is a random variable sampled uniformly, for each cylinder independently between $-a$ and $a$, with $a=0.01$. The pile is made the following way : the first horizontal line of cylinders is constructed so that neighbouring cylinders touch each other. On top of it, the second horizontal line is filled up in the same order : from left to right (Fig. 1a). Thus, except for the border elements, every new cylinder has three nearest neighbours already present in the packing. Among these three possible contacts, only two will be made. The probability that the three excluded volume circles cross at the same point vanishes since our distribution of $\varepsilon$ is continuous. Figure $1 \mathrm{~b}$ defines the three directions A, B and C. Two cases are encountered (Figs. 1c and d). In case (d), the location of the center 

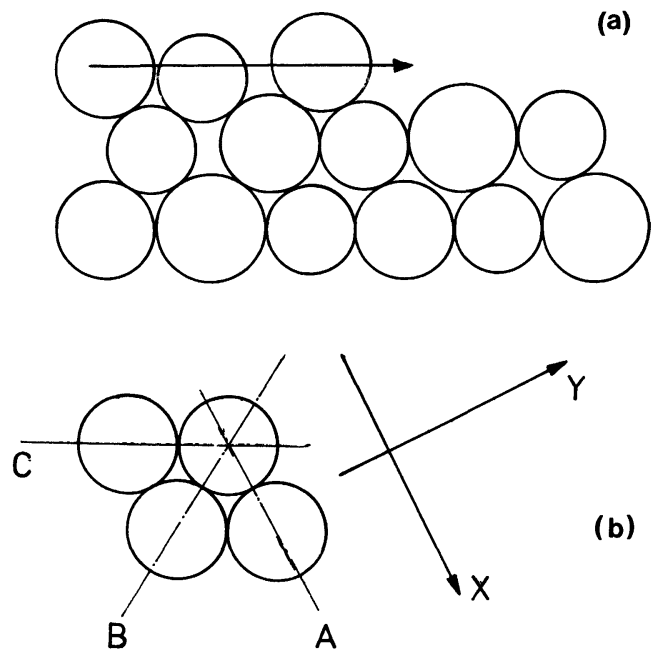

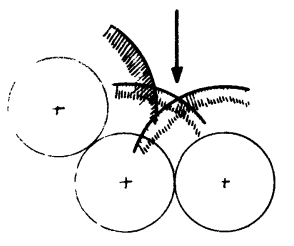

(c)

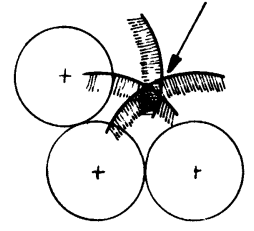

(d)
Fig. 1. - After having built a line of cylinders, the upper line is constructed starting from the left and proceeding towards the right (a). When one cylinder is added three of its neighbours are already present: $\mathrm{A}, \mathrm{B}$ and $\mathrm{C}(\mathrm{b})$. Their excluded volume can show two possible configurations: (c) and (d). The selected position (indicated by an arrow) is the one which minimizes the height. In (c) A and B contacts are established whereas in (d) A and C are selected.

of a new cylinder is given by the intersection of the excluded volume circles of $A$ and $C$. In case (c), two choices are possible in principle : contacts with $\mathrm{B}$ and C, or contacts with A and B. To remove the ambiguity, we will consider an additional condition by introducing an « infinitesimal » gravity field in the vertical direction. This will select only the contacts $A$ and $B$, which minimize the height of the new added cylinder. Thus, the A contacts will always be made whereas one and only one among $\mathrm{B}$ and $\mathrm{C}$ contacts will be made. However, we emphasize the fact that this is the only effect of gravity introduced in this study. The computer implementation of this algorithm is straightforward.

The precise definition of the way the piling is built in « time " is important. Not all directions are equivalent (Fig. 1a). In a physically more realistic model for piling, one could for example select at random the initial locations of the cylinders and then adjust them such as to obtain a stable situation. We believe however that in such a more realistic model each layer of cylinders will exhibit large domains of elements all of them with A-contacts, separated by domains with Bcontacts ; within each domain we expect a situation similar to the one we encounter in our model.
A triangular lattice structure (which would be the non-random case $\varepsilon=0$ ) is always present, although slightly altered, if the system is not too large. We define the « contact network » as the set of «bonds " of this triangular lattice for which there are contacts between cylinders.

\section{The structure of the contact network.}

Our problem can be decoupled in two directions : along the $X$ and the $Y$ axis (Fig. 1b). The contact network can be described as the union of two independent networks, one consisting of A-bonds and the other one being the set of B and C contacts. Thus the position of each site in these two networks is decomposed into two separate problems.

The A-contacts can be easily treated: in the $X$ direction, the location of a cylinder is determined by. the sum of the radii of the cylinders below, which is simply the sum of independent random variables. This distribution tends towards a Gaussian when a large number of A-contacts is added. In the contact network all lines of bonds parallel to $X$ will be present. If we disregard these lines we are left with the B and C contacts in the $Y$ direction.

The distribution of $\mathbf{B}$ and $\mathbf{C}$ contacts is non-trivial and exhibits interesting tree-like patterns (Fig. 2). In the limit of small disorder (small width of the distribution of the $\varepsilon$ ), we can neglect fluctuations in the angles between the contact-bonds. The coordinate, $Y_{i}$, of any cylinder $i$ in the $Y$ direction, or more precisely, its distance, $Z_{i}=Y_{i}-Y_{i}^{0}$, to the corresponding site in the

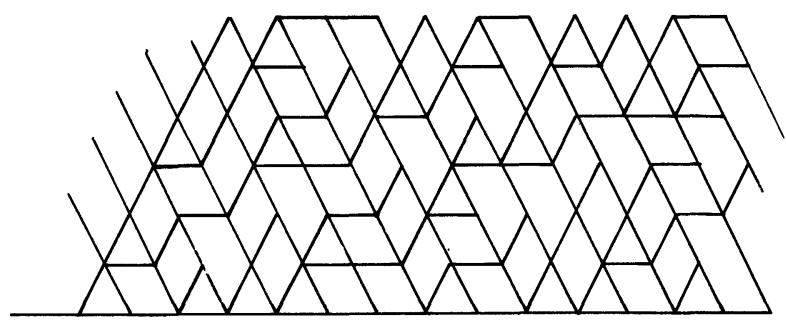

(a)

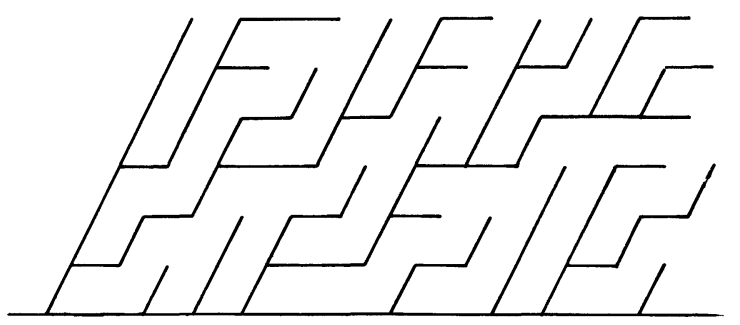

(b)

Fig. 2. - The contact network of a small sample is shown (a). When every line parallel to the $X$ axis are removed, (b), we see self-similar tree-like patterns « growing » from the bottom line. 
perfect triangular lattice, $Y_{i}^{0}$, can be calculated the following way.

On the sublattice of the $\mathrm{B}$ and $\mathrm{C}$ bonds (which is a tilted «oblique» square lattice) each site $i$ is given a continuously distributed random number $\varepsilon_{i}$ which represents the fluctuation in radius of the cylinder sitting on this site. Now we can compute $Z_{i}$ according to :

$$
\begin{aligned}
Z_{i}=\max \left(Z_{j}+(\sqrt{3} / 2) \varepsilon_{j}, Z_{k}+(\sqrt{3} / 2)\right. & \left.\varepsilon_{k}\right)+ \\
+ & (\sqrt{3} / 2) \varepsilon_{i}
\end{aligned}
$$

where $j$ and $k$ are the $\mathrm{B}$ and $\mathrm{C}$ neighbouring sites of $i$. The site, $j$ or $k$, which has the larger argument in equation (1) is in contact with $i$. We can therefore see that every site will be connected to one and only one bottom site on the left (B or C direction). Reciprocally, from each site a tree of contact bonds initiates which grows to the right and eventually dies off. The boundary condition in our piling is determined by the bottom row, which, as seen previously, assigns the value of a sum of independent random variables to $Z_{i}$. To understand better this stochastic growth process we can modify our boundary condition. If we assume that $Z_{i}=0$ on one line in the A direction, the direction of the growth is perpendicular to this basis line. In this situation we see that as the height increases some trees expand and others disappear until finally one tree spans the whole width of the system. Now we can ask at what rate do these trees grow, expand and die.

From a numerical simulation, we obtained a powerlaw decay of the number of independent trees, $N(h)$, as a function of height $h$, justified by the fact that no length scale exists, except that of the lattice. Namely (see Fig. 3)

$$
N(h) \propto h^{-x}
$$

where $x \simeq 0.62 \pm 0.02$. This result has been obtained from an average over 1000 realisations of $200 \times 200$ samples.

An approximate solution to this problem can be found in a simplified version : taking into account that one and only one bond connects a site with its left or left-down neighbour, but selecting these bonds at random, redefines another model, close to the previous one. (We neglect long-range correlations which come from the previous cylinder location in equation (1). For instance, if a large value of $\varepsilon$ has been chosen randomly for the site $i$ then the growth of the tree spanning from $i$ will be favoured in the original model). In the simplified model, an exact answer is available : let us put one particle on each of the sites of a line parallel to the basis (itself parallel to the $X$ axis) at a distance $h$ (height of the tree). Now, at one time step, every particle follows exactly one bond toward the basis. Each particle will thus accomplish a one-dimensional random walk in $X$ direction, where the time direction is in the negative $Y$ axis. Whenever two particles meet they stick forever

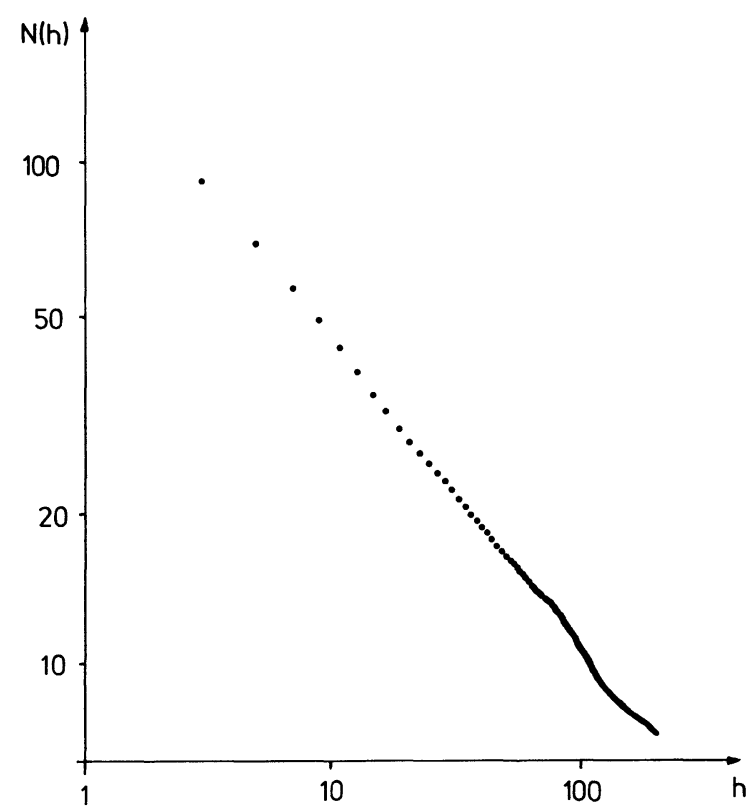

Fig. 3. - The growth of the trees as described through equation (1) has been simulated on $200 \times 200$ lattices. The direction of growth has been chosen perpendicular to the basis. We have plotted here on a log-log scale, the number of independent trees at a height $h, v s$. $h$. The data are averaged over 1000 samples.

and are regarded to be one single particle. The number of « trees » at height $h, N(h)$, is equal to the number of particles after a time $t=h$. Therefore the classical properties of the random walk allow us to conclude that

$$
N(h) \propto h^{-1 / 2}
$$

until it crosses over to $N(h)=1$ (for large $h$ ). A numerical simulation performed in this model confirmed the scaling relation of equation (3).

We note that in the limiting case of vanishing disorder $\varepsilon$ there is an abrupt change in the topological arrangement of contacts (e.g. the coordination number will jump from 4 when $\varepsilon>0$ to 6 when $\varepsilon=0$ ).

\section{Expansion of the lattice due to randomness.}

We have seen that the distribution of locations of the centers of the cylinders along the A direction tends towards a Gaussian distribution. In $Y$ direction one has the algorithm given by equation (1). The use of « $\max$ » functions selects biased values from the distribution of $\varepsilon$. Since $\varepsilon$ is uniformly distributed between $-a$ and $a$, the mean expansion of the total system height after $h$ steps is $(\sqrt{3} / 2) h a$. The numerical value of the last expression depends upon the particular choice of the distribution of $\varepsilon$, but the basic feature is that the expansion increases linearly with the height $h$ even if the average $\varepsilon$ is zero.

We point out that the trees form an angle $\pi / 6$ with the bottom line. Due to this inclination, the roots of the 
trees start from a « random walk » distribution of $Y$ coordinates, since the close packing of the first line gives the initial contribution in that direction. So, for a given height, the mean vertical expansion increases as $\sqrt{L}$ where $L$ is the width of the piling. Our numerical results are shown in figure 4 and confirm this theoretical prediction when $L$ is large.

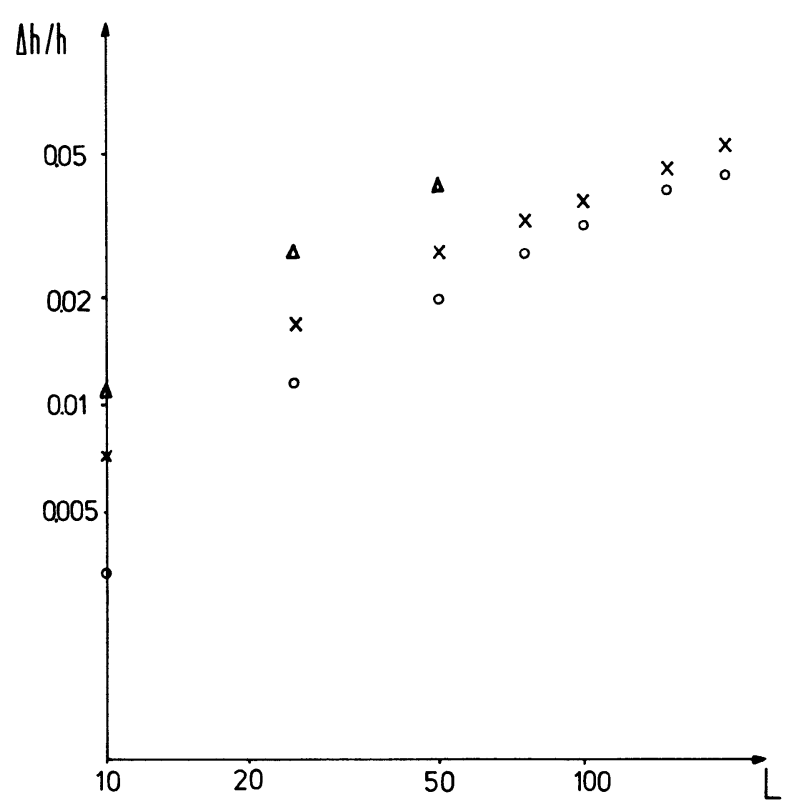

Fig. 4. - For the original piling problem, we show on a log$\log$ plot the mean vertical expansion as a function of the width of the system, for a height of $5(\mathrm{X})$ and $11(\mathrm{O})$ and a uniform distribution of $\varepsilon$ between $-a$ and $a .(a=0.01)$. The symbol $(\Delta)$ refers to a bimodal distribution: $\varepsilon= \pm a$ with equal probability 0.5 and a height of 5 .

The fact that we obtain an ever increasing expansion shows the limitations of our algorithm. At large distances from the origin there will be holes so large that entire cylinders could fit into them. We cannot expect that the underlying triangular lattice free of defects will survive over very large distances. However we believe that over intermediate length scales this model is acceptable. We will see in the companion article [4] that obtaining a piling by relaxing it to a zero-force limit is a difficult task to perform (the computer time needed is very long in this limit), but it gives more realistic expansion factors on large scales.

Using the same program, we also computed the distribution of distances separating two nearest neighbours when there is no contact. We obtained an exponential distribution as shown in figure 5 .

Our problem resembles that of rigidity percolation [7] if one replaces a contact by a central-force spring. Moreover, in our piling, the fraction of contacts is exactly $2 / 3$ of the bonds of the triangular lattice, close

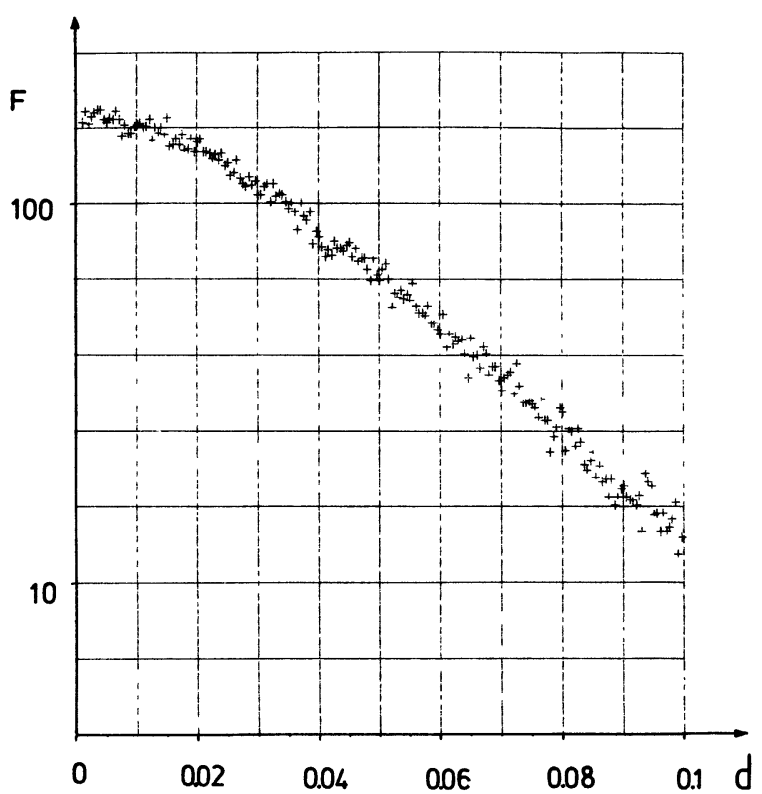

Fig. 5. - Distribution of the distances between adjacent cylinders on a semi-log plot : log of the frequency (arbitrary units) $v s$. distance. These data are obtained from 300 samples of height 5 and width 200 .

to the rigidity percolation threshold [8] $0.655 \pm 0.005$. This agreement is not too surprising since the effective medium approximation predicts also a threshold [9] of $2 / 3$. The analogy with the rigidity percolation problem is limited : our algorithm introduces correlations which are absent in rigidity percolation. However, one common feature is the vector nature of both problems (discussed in detail in Ref. [10] for rigidity percolation).

\section{Conclusion.}

Analysing the generated contact network, we found a rich structure of self-similar tree-like patterns, as well as strong effects depending on the system size. It would be interesting to search experimentally for the existence of such contacts and to try to find the range over which our model describes correctly the situation. The limitations of our model (and its natural extension) for very large lattices and/or large $\varepsilon$ is the problem of strongly disordered packing [6]. We also want to emphasize that contact (bond), instead of composition (site), is probably the more relevant basic concept to analyse transport and mechanical properties of a disordered packing of objects.

\section{Acknowledgments.}

It is a pleasure to acknowledge fruitful discussions with E. Guyon, D. Bideau, A. Gervois and J. P. Troadec and their comments on the manuscript. 


\section{References}

[1] Kantor, Y. and Webman, I., Phys. Rev. Lett. 52 (1984) 1891.

FEng, S. and SEN, P. N., Phys. Rev. Lett. 52 (1984) 216.

Roux, S. and Guyon, E., J. Physique Lett. 46 (1985) L-999

[2] Travers, T., Bideau, D., Gervois, A., Troadec, J. P. and Messager, J. C., J. Phys. A 19 L1033.

[3] Timoshenko, S., Théorie de l'élasticité, Ch. Béranger (Paris and Lille) 1948 in particular section 107.

[4] Stauffer, D., Herrmann, H. J. and Roux, S., following article.

[5] Blumenfeld, R. and Aharony, A., J. Phys. A 18 (1985) L443.
De Arcangelis, L., Coniglio, A. and Redner, S., J. Phys. A 18 (1985) L805.

Gefen, Y., Shih, W. H., Laibowitz, R. B. and Viggiano, J. M., Phys. Rev. Lett. 57 (1986) 3097.

Roux, S., Herrmann, H., in preparation.

[6] Bideau, D., Gervois, A., Oger, L. and Troadec, J. P., preprint.

[7] Feng, S. and Sen, P. N., Phys. Rev. Lett. 52 (1984) 216.

[8] Lemieux, M. A., Breton, P. and Tremblay, A. M. S., J. Physique Lett. 46 (1985) L-1.

[9] Feng, S., Thorpe, M. F. and Garboczi, E., Phys. Rev. B 31 (1985) 276.

[10] Day, A. R., Tremblay, R. R. and Tremblay, A. M. S., Phys. Rev. Lett. 56 (1986) 2501. 